\title{
A Note on the Performance of Biased Estimators with Autocorrelated Errors
}

\author{
Gargi Tyagi and Shalini Chandra \\ Department of Mathematics \& Statistics, Banasthali University, Rajasthan 304022, India \\ Correspondence should be addressed to Gargi Tyagi; tyagi.gargi@gmail.com
}

Received 31 July 2016; Revised 20 November 2016; Accepted 7 December 2016; Published 30 January 2017

Academic Editor: Weimin Han

Copyright (C) 2017 Gargi Tyagi and Shalini Chandra. This is an open access article distributed under the Creative Commons Attribution License, which permits unrestricted use, distribution, and reproduction in any medium, provided the original work is properly cited.

\begin{abstract}
It is a well-established fact in regression analysis that multicollinearity and autocorrelated errors have adverse effects on the properties of the least squares estimator. Huang and Yang (2015) and Chandra and Tyagi (2016) studied the PCTP estimator and the $r-(k, d)$ class estimator, respectively, to deal with both problems simultaneously and compared their performances with the estimators obtained as their special cases. However, to the best of our knowledge, the performance of both estimators has not been compared so far. Hence, this paper is intended to compare the performance of these two estimators under mean squared error (MSE) matrix criterion. Further, a simulation study is conducted to evaluate superiority of the $r-(k, d)$ class estimator over the PCTP estimator by means of percentage relative efficiency. Furthermore, two numerical examples have been given to illustrate the performance of the estimators.
\end{abstract}

\section{Introduction}

Let us consider a linear regression model as

$$
y=X \beta+u,
$$

where $y$ is an $n \times 1$ vector of observations on dependent variable, $X$ is an $n \times p$ full column rank matrix of observations on $p$ explanatory variables, $\beta$ is a $p \times 1$ vector of unknown regression coefficients, and $u$ is an $n \times 1$ vector of disturbance term with mean vector 0 and covariance matrix $\sigma^{2} I_{n}$.

Ordinary least squares estimator (OLSE) is one of the most widely used estimator for $\beta$, given as

$$
\widehat{\beta}=\left(X^{\prime} X\right)^{-1} X^{\prime} y \text {. }
$$

In the presence of multicollinearity among explanatory variables, OLSE becomes unstable and shows undesirable properties, such as inflated variance, wide confidence intervals which leads to wrong inferences and sometimes it even produces wrong signs of the estimates.

Numerous alternative methods of estimation have been designed to lower the effects of multicollinearity in literature.
For instance, Stein [1] proposed stein estimator; Hoerl and Kennard $[2,3]$ introduced the technique of ordinary ridge regression estimator (ORRE); Massy [4] suggested principal component regression estimator (PCRE) to deal with the problem. Several authors combined two techniques of estimation in the hope that the combination will contain the advantages of the both. Baye and Parker [5] gave $r-$ $k$ class estimator by combining the PCRE and the ORRE, which includes the OLSE, ORRE, and PCRE as special cases. Nomura and Ohkubo [6] obtained conditions for dominance of the $r-k$ class estimator over its special cases under mean squared error (MSE) criterion. Liu [7] gave an estimator by combining the advantages of the stein and ORRE, known as Liu estimator (LE). Kaçiranlar and Sakallığlu [8] proposed $r-d$ class estimator which is a combination of the LE and PCRE and showed the superiority of the $r-d$ class estimator over the OLSE, LE, and PCRE. Özkale and Kaçıranlar [9] proposed two-parameter estimator (TPE) by utilizing the advantages of the ORRE and LE and obtained necessary and sufficient condition for dominance of the TPE over the OLSE in MSE matrix sense. Further, Yang and Chang [10] also combined the ORR and Liu estimator in a different way and introduced an another two-parameter estimator (ATPE) and 
derived necessary and sufficient conditions for superiority of the ATPE over OLSE, ORRE, LE, and TPE under MSE matrix criterion. Özkale [11] put forward a general class of estimators, $r-(k, d)$ class estimator which is a mingle of the TPE [9] and PCRE; they evaluated the performance of the $r-(k, d)$ class estimator under MSE criterion. Chang and Yang [12] suggested another general class of estimators by merging the PCRE and ATPE [10] named as principal component twoparameter estimator (PCTPE) and analyzed its performance under MSE matrix sense.

In applied work, it is quite common to have autocorrelation in error terms; that is, $\operatorname{cov}(u)=\sigma^{2} \Omega$, where $\Omega$ is a known symmetric positive definite (p.d.) $n \times n$ matrix and it is well known to statisticians that autocorrelated errors reduce the efficiency of the OLSE. Now, since $\Omega$ is a symmetrical positive definite matrix there exists an orthogonal matrix $E$ such that $E E^{\prime}=\Omega$. On premultiplying model (1) by $E^{-1}$, we have

$$
\begin{gathered}
E^{-1} y=E^{-1} X \beta+E^{-1} u \\
y^{*}=X^{*} \beta+u^{*} .
\end{gathered}
$$

Note that $E\left(u^{*}\right)=0$ and $\operatorname{cov}\left(u^{*}\right)=\sigma^{2} I$.

To overcome the effect of autocorrelated errors, Aitken [13] proposed the generalized least squares estimator (GLSE) for $\beta$ in (1) which can be obtained by applying least squares technique in model (3) as

$$
\widehat{\beta}_{\mathrm{GLS}}=\left(X^{\prime} \Omega^{-1} X\right)^{-1} X^{\prime} \Omega^{-1} y .
$$

It has been observed that the problem of autocorrelation and multicollinearity arise simultaneously in several cases. Keeping this in mind, a good amount of literature has been devoted to study these problems simultaneously by Trenkler [14], Firinguetti [15], G. M. Bayhan and M. Bayhan [16], Alheety and Kibria [17], Özkale [18], Güler and Kaçiranlar [19], Alkhamisi [20], Yang and Wu [21], Eledum and Alkhalifa [22], Şiray [23], and Chandra and Sarkar [24], to name a few.

Further, to define the estimators, let $T=\left(t_{1}, t_{2}, \ldots, t_{p}\right)$ be a $p \times p$ orthogonal matrix with $T^{\prime} X^{\prime} \Omega^{-1} X T=\Lambda=$ $\operatorname{diag}\left(\lambda_{1}, \lambda_{2}, \ldots, \lambda_{p}\right)$, where $\Lambda$ is a $p \times p$ diagonal matrix of eigenvalues of $X^{\prime} \Omega^{-1} X$ matrix such that $\lambda_{1} \geq \lambda_{2} \geq$ $\cdots \geq \lambda_{p}$. Now, let $T_{r}=\left(t_{1}, t_{2}, \ldots, t_{r}\right)$ be $p \times r$ orthogonal matrix after deleting last $p-r$ columns from $T$ matrix, where $r \leq p$. Thus, $T_{r}^{\prime} X^{\prime} \Omega^{-1} X T_{r}=\Lambda_{r}$ where $\Lambda_{r}=$ $\operatorname{diag}\left(\lambda_{1}, \lambda_{2}, \ldots, \lambda_{r}\right)$ and $T_{p-r}^{\prime} X^{\prime} \Omega^{-1} X T_{p-r}=\Lambda_{p-r}$, where $\Lambda_{p-r}=\operatorname{diag}\left(\lambda_{r+1}, \lambda_{2}, \ldots, \lambda_{p}\right)$. Also, $T^{\prime} T=T_{r}^{\prime} T_{r}+T_{p-r}^{\prime} T_{p-r}$.

Chandra and Tyagi [25] modified the $r-(k, d)$ class estimator [11] to address multicollinearity and autocorrelated errors simultaneously, which is expressed as

$$
\begin{aligned}
& \widehat{\beta}_{r}(k, d)=T_{r}\left(T_{r}^{\prime} X^{\prime} \Omega^{-1} X T_{r}+k I_{r}\right)^{-1} \\
& \cdot\left(T_{r}^{\prime} X^{\prime} \Omega^{-1} X T_{r}\right)^{-1}\left(T_{r}^{\prime} X^{\prime} \Omega^{-1} X T_{r}+k d I_{r}\right) \\
& \cdot T_{r}^{\prime} X^{\prime} \Omega^{-1} y=T_{r} S_{r}(k)^{-1} \Lambda_{r}^{-1} S_{r}(k d) T_{r}^{\prime} X^{\prime} \Omega^{-1} y,
\end{aligned}
$$

where $S_{r}(q)=\Lambda_{r}+q I_{r}, q=1, k, d, k d$.
Huang and Yang [26] proposed PCTP estimator in the presence of autocorrelated errors as

$$
\begin{aligned}
& \tilde{\beta}_{r}(k, d)=T_{r}\left(T_{r}^{\prime} X^{\prime} \Omega^{-1} X T_{r}+I_{r}\right)^{-1} \\
& \cdot\left(T_{r}^{\prime} X^{\prime} \Omega^{-1} X T_{r}+d I_{r}\right)\left(T_{r}^{\prime} X^{\prime} \Omega^{-1} X T_{r}+k I_{r}\right)^{-1} \\
& \cdot T_{r}^{\prime} X^{\prime} \Omega^{-1} y=T_{r} S_{r}(1)^{-1} S_{r}(d) S_{r}(k)^{-1} T_{r}^{\prime} X^{\prime} \Omega^{-1} y, \\
& k>0,0<d<1 .
\end{aligned}
$$

Some other biased estimators in the presence of multicollinearity and autocorrelation can be obtained as special cases. $\widehat{\beta}_{r}(k, 0)=\widetilde{\beta}_{r}(k, 1)=\widehat{\beta}_{r}(k)$ is the $r-k$ class estimator proposed by Şiray et al. [23]; $\widehat{\beta}_{p}(0, d)=\widetilde{\beta}_{p}(1,0)=\widehat{\beta}_{\mathrm{GLS}}$ is the GLSE by Aitken [13]; $\widehat{\beta}_{p}(k, 0)=\widetilde{\beta}_{p}(k, 1)=\widehat{\beta}(k)$ is the ridge regression estimator (RRE) given by Trenkler [14] and so forth. The special cases of the estimators have been compared with the $r-(k, d)$ class estimator and the PCTP estimator by Chandra and Tyagi [25] and Huang and Yang [26], respectively. Hence, this paper focuses on the comparison of the performance of the two general estimators.

Further, the rest of the paper is organized as follows: the necessary and sufficient condition for dominance of the PCTP estimator over the $r-(k, d)$ class estimator under the MSE matrix criterion has been derived in Section 2. Section 3 is devoted to simulation study to compare these estimators under MSE criterion. Some methods of selection of the unknown biasing parameters have been given in Section 4. Section 5 includes two numerical examples. Finally, the paper is summed up in Section 6 with some concluding remarks.

\section{MSE Matrix Comparison of $\widehat{\beta}_{r}(k, d)$ and $\widetilde{\beta}_{r}(k, d)$}

The MSE matrix criterion is a strong and one of the most widely used criteria for comparison of the estimators. Let $\breve{\beta}$ be an estimator of $\beta$; then the expression for the MSE matrix is given as

$$
\begin{aligned}
M(\breve{\beta}) & =E\left[(\breve{\beta}-\beta)(\breve{\beta}-\beta)^{\prime}\right] \\
& =\operatorname{cov}(\breve{\beta})-\operatorname{Bias}(\breve{\beta}) \operatorname{Bias}(\breve{\beta})^{\prime},
\end{aligned}
$$

where $\operatorname{cov}(\breve{\beta})$ and $\operatorname{Bias}(\breve{\beta})$ are the covariance matrix and bias vector of $\breve{\beta}$.

From (5) and (6), the covariance matrices and bias vectors of the $r-(k, d)$ estimator and PCTP estimator can be obtained as

$$
\operatorname{cov}\left(\widehat{\beta}_{r}(k, d)\right)=\sigma^{2} T_{r} S_{r}(k)^{-1} S_{r}(k d) \Lambda_{r}^{-1} S_{r}(k d)
$$

$$
\text { - } S_{r}(k)^{-1} T_{r}^{\prime}
$$




$$
\begin{aligned}
& \operatorname{cov}\left(\tilde{\beta}_{r}(k, d)\right)=\sigma^{2} T_{r} S_{r}(1)^{-1} S_{r}(d) S_{r}(k)^{-1} \Lambda_{r} S_{r}(k)^{-1} \\
& \cdot S_{r}(d) S_{r}(1)^{-1} T_{r}^{\prime} \\
& \operatorname{Bias}\left(\widehat{\beta}_{r}(k, d)\right) \\
&=-\left(k(1-d) T_{r} S_{r}(k)^{-1} T_{r}^{\prime}+T_{p-r} T_{p-r}^{\prime}\right) \beta \\
&=-T B_{1} T^{\prime} \beta
\end{aligned}
$$

$$
\begin{aligned}
\operatorname{Bias} & \left(\widetilde{\beta}_{r}(k, d)\right) \\
= & \left(T_{r} S_{r}(1)^{-1} S_{r}(d) S_{r}(k)^{-1} \Lambda_{r} T_{r}^{\prime}-I_{p}\right) \beta \\
= & -T B_{2} T^{\prime} \beta,
\end{aligned}
$$

where $\quad B_{1}=\left(\begin{array}{cc}k(1-d) S_{r}(k)^{-1} & 0 \\ 0 & I_{p-r}\end{array}\right)$ and $B_{2}=$
$\left(\begin{array}{c}S_{r}(1)^{-1}\left(\Lambda_{r}(1+k-d)+k I_{r}\right) S_{r}(k)^{-1} \\ 0 \\ I_{p-r}\end{array}\right)$.

Thus, the MSE matrices of the estimators can be given as

$$
\begin{aligned}
& M\left(\widehat{\beta}_{r}(k, d)\right)=\sigma^{2} T_{r} S_{r}(k)^{-1} S_{r}(k d) \Lambda_{r}^{-1} S_{r}(k d) \\
& \quad \cdot S_{r}(k)^{-1} T_{r}^{\prime}+T B_{1} T^{\prime} \beta \beta^{\prime} T B_{1} T^{\prime} \\
& M\left(\widetilde{\beta}_{r}(k, d)\right)=\sigma^{2} T_{r} S_{r}(1)^{-1} S_{r}(d) S_{r}(k)^{-1} \Lambda_{r} S_{r}(k)^{-1} \\
& \quad \cdot S_{r}(d) S_{r}(1)^{-1} T_{r}^{\prime}+T B_{2} T^{\prime} \beta \beta^{\prime} T B_{2} T^{\prime} .
\end{aligned}
$$

To compare the performance of these estimators, the difference of the MSE matrices can be obtained as

$$
\begin{gathered}
\Delta_{M}=M\left(\widehat{\beta}_{r}(k, d)\right)-M\left(\widetilde{\beta}_{r}(k, d)\right)=\sigma^{2} T_{r} S_{r}(k)^{-1} \\
\cdot S_{r}(k d) \Lambda_{r}^{-1} S_{r}(k d) S_{r}(k)^{-1} T_{r}^{\prime}+T B_{1} T^{\prime} \beta \beta^{\prime} T B_{1} T^{\prime} \\
-T_{r} S_{r}(1)^{-1} S_{r}(d) S_{r}(k)^{-1} \Lambda_{r} S_{r}(k)^{-1} S_{r}(d) S_{r}(1)^{-1} \\
\cdot T_{r}^{\prime}-T B_{2} T^{\prime} \beta \beta^{\prime} T B_{2} T^{\prime}=D+a_{1} a_{1}^{\prime}-a_{2} a_{2}^{\prime},
\end{gathered}
$$

where $D=\sigma^{2} T_{r} S_{r}(k)^{-1}\left[S_{r}(k d) \Lambda_{r}^{-1} S_{r}(k d) \quad-\right.$ $\left.S_{r}(1)^{-1} S_{r}(d) \Lambda_{r} S_{r}(d) S_{r}(1)^{-1}\right] S_{r}(k)^{-1} T_{r}^{\prime}, \quad a_{1}=T B_{1} T^{\prime} \beta$, $a_{2}=T B_{2} T^{\prime} \beta$.

On further simplification, $D$ can be written as

$$
\begin{aligned}
D= & \sigma^{2} T_{r} S_{r}(k)^{-1} S_{r}(1)^{-1}\left((k d+1-d) I_{r}+k d \Lambda_{r}^{-1}\right) \\
& \cdot\left(2 \Lambda_{r}^{2}+(k d+d+1) \Lambda_{r}+k d I_{r}\right) S_{r}(1)^{-1} \\
& \cdot S_{r}(k)^{-1} T_{r}^{\prime} .
\end{aligned}
$$

It is easy to note that $D$ is positive definite. For the convenience of the derivation of the dominance conditions, we state the following Lemma.
Lemma 1. Assume that $\widehat{\beta}_{j}=A_{j} y, j=1,2$, are two competing linear estimators of $\beta$. Suppose that $D=\operatorname{cov}\left(\widehat{\beta}_{1}\right)-\operatorname{cov}\left(\widehat{\beta}_{2}\right)>0$, where $\operatorname{cov}\left(\widehat{\beta}_{j}\right), j=1,2$, denotes the covariance matrix of $\widehat{\beta}_{j}$. Then $\Delta\left(\widehat{\beta}_{1}, \widehat{\beta}_{2}\right)=M\left(\widehat{\beta}_{1}\right)-M\left(\widehat{\beta}_{2}\right) \geq 0$ if and only if $d_{2}^{\prime}(D+$ $\left.d_{1} d_{1}^{\prime}\right)^{-1} d_{2} \leq 1$, where $d_{j}$ denote the bias vector of $\widehat{\beta}_{j}$.

From the expressions in (5) and (6), it is easy to verify that the $r-(k, d)$ class estimator and the PCTP estimator can be written as $\widehat{\beta}_{r}(k, d)=A_{1} \widehat{\beta}_{\mathrm{GLS}}$ and $\widetilde{\beta}_{r}(k, d)=$ $A_{2} \widehat{\beta}_{\text {GLS }}$, where $A_{1}=T_{r} S_{r}(k)^{-1} S_{r}(k d) T_{r}^{\prime}$ and $A_{2}=$ $T_{r} S_{r}(1)^{-1} \Lambda_{r} S_{r}(d) S_{r}(k)^{-1} T_{r}^{\prime}$. Further, it is evident from (12) that $D=\operatorname{cov}\left(\widehat{\beta}_{r}(k, d)\right)-\operatorname{cov}\left(\widetilde{\beta}_{r}(k, d)\right)$ is a positive definite matrix. Thus from the above lemma, $\Delta_{M} \geq 0$ if and only if

$$
a_{2}^{\prime}\left(D+a_{1} a_{1}^{\prime}\right)^{-1} a_{2} \leq 1 .
$$

Hence, the comparison under MSE matrix can be concluded in the following theorem.

Theorem 2. The PCTP estimator dominates the $r-(k, d)$ class estimator in MSE matrix sense if and only if $a_{2}^{\prime}\left(D+a_{1} a_{1}^{\prime}\right)^{-1} a_{2} \leq$ 1.

\section{Selection of $k$ and $d$}

It is an important problem to find optimum value of the biasing parameters. A general approach to select an optimum value of the biasing parameters is to minimize the scalar MSE of the estimator.

3.1. For $\widehat{\beta}_{r}(k, d)$. The scalar MSE of the $r-(k, d)$ class estimator can be obtained by taking trace of the MSE matrix in (9), which is given as

$$
\begin{aligned}
m_{1}(r, k, d)= & \sum_{i=1}^{r} \frac{\sigma^{2}\left(\lambda_{i}+k d\right)^{2}+k^{2}(1-d)^{2} \lambda_{i} \alpha_{i}^{2}}{\lambda_{i}\left(\lambda_{i}+k\right)^{2}} \\
& +\sum_{i=r+1}^{p} \alpha_{i}^{2}
\end{aligned}
$$

where $\alpha_{i}=i$ th component of $\alpha=T^{\prime} \beta$. The optimum value of $k(d)$ for a fixed $d(k)$ and $r$ can be obtained by differentiating $m_{1}(r, k, d)$ with respect to $k(d)$ and equating it to zero. Further, first derivative of $m_{1}(r, k, d)$ with respect to $k$ for fixed $r$ and $d$ is obtained as

$$
\begin{aligned}
& \frac{\partial m_{1}(r, k, d)}{\partial k} \\
& =\sum_{i=1}^{r} \frac{2(1-d) \lambda_{i}\left\{\left(k \lambda_{i} \alpha_{i}^{2}-\sigma^{2}\right)-k d\left(\sigma^{2}+\lambda_{\mathrm{i}} \alpha_{i}^{2}\right)\right\}}{\left(\lambda_{i}+k\right)^{3}}
\end{aligned}
$$


On equating to zero, we get the value of $k$ for the $r-(k, d)$ class estimator as

$$
k_{1}(\text { say })=\frac{\sigma^{2}}{\left(\alpha_{i}^{2}-d\left(\sigma^{2} / \lambda_{i}+\alpha_{i}^{2}\right)\right)}, \quad \forall i=1,2, \ldots, r .
$$

By taking harmonic mean as suggested by Hoerl et al. [27] and arithmetic mean and geometric mean [28] of the values in (16), we propose the following estimators:

$$
\begin{aligned}
& k_{1_{\mathrm{HM}}}=\frac{r \sigma^{2}}{\sum_{i=1}^{r}\left(\alpha_{i}^{2}-d\left(\sigma^{2} / \lambda_{i}+\alpha_{i}^{2}\right)\right)}, \\
& k_{1_{\mathrm{AM}}}=\frac{1}{r} \sum_{i=1}^{r} \frac{\sigma^{2}}{\left(\alpha_{i}^{2}-d\left(\sigma^{2} / \lambda_{i}+\alpha_{i}^{2}\right)\right)}, \\
& k_{1_{\mathrm{GM}}}=\frac{\sigma^{2}}{\left(\prod_{i=1}^{r}\left(\alpha_{i}^{2}-d\left(\sigma^{2} / \lambda_{i}+\alpha_{i}^{2}\right)\right)\right)^{1 / r}} .
\end{aligned}
$$

Further, the positiveness of $k_{1}$ can be ensured when we have $d<\min _{i=1,2, \ldots, r}\left\{\alpha_{i}^{2} /\left(\sigma^{2} / \lambda_{i}+\alpha_{i}^{2}\right)\right\}=d_{1}$ (say). It can be noted that when $d=\min _{i=1,2, \ldots, r}\left\{\alpha_{i}^{2} /\left(\sigma^{2} / \lambda_{i}+\alpha_{i}^{2}\right)\right\}, k_{1_{\mathrm{GM}}}$ is not defined and $k_{1_{\mathrm{GM}}}$ will give zero. This way we can choose a value of $d$ satisfying $d<d_{1}$ and further the value of $k$ is obtained by replacing $d$ in (17).
Alternatively, for fixed $r$ and $k$, the optimum value of $d$ for the $r-(k, d)$ class estimator by minimizing $m_{1}(r, k, d)$ with respect to $d$ is obtained as

$$
d_{1_{\mathrm{opt}}}=\frac{\sum_{i=1}^{r}\left(\left(k \alpha_{i}^{2}-\sigma^{2}\right) /\left(\lambda_{i}+k\right)^{2}\right)}{\sum_{i=1}^{r}\left(k\left(\sigma^{2}+\lambda_{i} \alpha_{i}^{2}\right) / \lambda_{i}\left(\lambda_{i}+k\right)^{2}\right)}
$$

Clearly, $d_{1_{\text {opt }}}$ is positive when $k>\sigma^{2} / \alpha_{\text {min }}^{2}$. Hence, we can choose a value of $k>\sigma^{2} / \alpha_{\text {min }}^{2}$ and making use of this value we can find optimum value $d_{1_{\text {opt }}}$.

3.2. For $\widetilde{\beta}_{r}(k, d)$. The scalar MSE of the PCTP estimator obtained by taking trace of the MSE matrix in (10) is given as

$$
\begin{aligned}
& m_{2}(r, k, d) \\
& =\sum_{i=1}^{r} \frac{\sigma^{2} \lambda_{i}\left(\lambda_{i}+d\right)^{2}+\left(\lambda_{i}(1+k-d)+k\right)^{2} \alpha_{i}^{2}}{\left(\lambda_{i}+1\right)^{2}\left(\lambda_{i}+k\right)^{2}} \\
& \quad+\sum_{i=r+1}^{p} \alpha_{i}^{2} .
\end{aligned}
$$

The first order derivative of $m_{2}(r, k, d)$ is obtained as

$$
\frac{\partial m_{2}(r, k, d)}{\partial k}=\sum_{i=1}^{r} \frac{\left(2\left(\lambda_{i}+1\right)\left(\lambda_{i}+k\right)\left(\lambda_{i}(1+k-d)+k\right) \alpha_{i}^{2}-2 \lambda_{i}\left(\lambda_{i}+d\right)^{2} \sigma^{2}-2\left(\lambda_{i}(1+k-d)+k\right)^{2} \alpha_{i}^{2}\right)}{\left(\lambda_{i}+1\right)^{2}\left(\lambda_{i}+k\right)^{3}} .
$$

The optimum value of $k$ for the PCTP estimator is obtained as

$$
k_{2}=\frac{\left(\lambda_{i}+d\right) \sigma^{2}-(1-d) \lambda_{i} \alpha_{i}^{2}}{\left(\lambda_{i}+1\right) \alpha_{i}^{2}}, \quad \forall i=1,2, \ldots, r
$$

Since $k_{2}$ depends on $i$, following Hoerl et al. [27] and Kibria [28], we propose the following estimators:

$$
\begin{aligned}
& k_{2_{\mathrm{HM}}} \\
& =\frac{r}{\sum_{i=1}^{r}\left(\left(\left(\lambda_{i}+d\right) \sigma^{2}-(1-d) \lambda_{i} \alpha_{i}^{2}\right) /\left(\lambda_{i}+1\right) \alpha_{i}^{2}\right)}, \\
& k_{2_{\mathrm{AM}}}=\frac{1}{r} \sum_{i=1}^{r}\left(\frac{\left(\lambda_{i}+d\right) \sigma^{2}-(1-d) \lambda_{i} \alpha_{i}^{2}}{\left(\lambda_{i}+1\right) \alpha_{i}^{2}}\right), \\
& k_{2_{\mathrm{GM}}}=\left(\prod_{i=1}^{r} \frac{\left(\lambda_{i}+d\right) \sigma^{2}-(1-d) \lambda_{i} \alpha_{i}^{2}}{\left(\lambda_{i}+1\right) \alpha_{i}^{2}}\right)^{1 / r} .
\end{aligned}
$$

Further, the positiveness of $k_{2}$ can be ensured when we have $d>\max _{i=1,2, \ldots, r}\left\{\left(\alpha_{i}^{2}-\sigma^{2}\right) /\left(\sigma^{2} / \lambda_{i}+\alpha_{i}^{2}\right)\right\}=d_{2}$. This way we can chose a value of $d$ satisfying $d>d_{2}$ for the PCTP estimator and the value of $k$ is then obtained by replacing $d$ in (22).
Alternatively, for fixed $r$ and $k$, the optimum values of $d$ for the PCTP estimator by minimizing $m_{2}(r, k, d)$ with respect to $d$ are obtained as

$$
\begin{aligned}
& d_{2_{\text {opt }}} \\
& =\frac{\sum_{i=1}^{r}\left(\left(k\left(\lambda_{i}+1\right) \alpha_{i}^{2}-\lambda_{i}\left(\sigma^{2}-\alpha_{i}^{2}\right)\right) /\left(\lambda_{i}+1\right)^{2}\left(\lambda_{i}+k\right)^{2}\right)}{\sum_{i=1}^{r}\left(\left(\sigma^{2}+\lambda_{i} \alpha_{i}^{2}\right) /\left(\lambda_{i}+1\right)^{2}\left(\lambda_{i}+k\right)^{2}\right)} .
\end{aligned}
$$

When $\sigma^{2}-\alpha_{i}^{2}>0$ for some $i=1,2, \ldots, r, 0<d_{2_{\text {opt }}}<1$ for

$$
\max _{i=1,2, \ldots, r}\left\{\frac{\lambda_{i}\left(\sigma^{2}-\alpha_{i}^{2}\right)}{\left(\lambda_{i}+1\right) \alpha_{i}^{2}}\right\}<k<\frac{\sigma^{2}}{\alpha_{\max }^{2}} .
$$

When $\sigma^{2}-\alpha_{i}^{2}<0$ for all $i=1,2, \ldots, r, 0<d_{2_{\text {opt }}}<1$ for $0<k<\sigma^{2} / \alpha_{\max }^{2}$.

Further, the values of $k$ and $d$ can be easily obtained by replacing the unknown parameters $\sigma^{2}$ and $\alpha$ with their unbiased estimators. 


\section{Monte Carlo Study}

In this section, we will evaluate the performance of the estimators through Monte Carlo simulation. Following McDonald and Galarneau [29] and Gibbons [30], X matrix has been generated as follows:

$$
\begin{aligned}
x_{i j}=\left(1-\gamma^{2}\right)^{1 / 2} z_{i j}+\gamma z_{i p+1}, & \\
& \quad i=1,2, \ldots, n, j=1,2, \ldots, p,
\end{aligned}
$$

where $z_{i j}$ are generated from standard normal pseudorandom numbers and $x_{i j}$ 's are generated such that the correlation between any pair of $X$-variables is $\gamma^{2}$. In this study, we consider the values of $\gamma$ to be $0.90,0.95$, and 0.99 . Following McDonald and Galarneau [29], Gibbons [30], Kibria [28], and others, $\beta$ has been chosen as the normalized eigenvector corresponding to the largest eigenvalue of the $X^{\prime} X$ matrix. The dependent variable $y$ is obtained by

$$
y=X \beta+u
$$

Following Firinguetti [15], Judge et al. [31], and Chandra and Sarkar [24], $u$ are generated from AR(1) process as

$$
u_{i}=\rho u_{i-1}+e_{i}, \quad i=1,2, \ldots, n,
$$

where $e_{i}$ are independent normal pseudorandom numbers with mean 0 and variance $\sigma_{e}^{2}$ and $\rho$ is autoregressive coefficient such that $|\rho|<1$. The covariance matrix $\Omega$ for $\operatorname{AR}(1)$ errors is given by

$$
\Omega=\left(\omega_{i j}\right)_{n \times n}, \quad \omega_{i j}=\sigma^{2} \rho^{|i-j|}, \text { where } \sigma^{2}=\frac{\sigma_{e}^{2}}{1-\rho^{2}} .
$$

The value of $r$ is decided by a scree plot which is drawn between eigenvalues and components (see Johnson and Wichern [32]). In this simulation we chose $n=20,50,100$, $p=5,10, \sigma_{e}^{2}=0.1,1,10, \rho=0,0.3,0.9, k=0.1,0.5,0.9,2$, and $d=0.1,0.5,0.9$. Then the experiment is repeated 2000 times by generating errors in every repetition and estimated MSE (EMSE) is calculated by the following formula:

$$
\operatorname{EMSE}(\widehat{\beta})=\frac{1}{2000} \sum_{i=1}^{2000}\left(\widehat{\beta}_{(i)}-\beta\right)^{\prime}\left(\widehat{\beta}_{(i)}-\beta\right),
$$

where $\widehat{\beta}_{(i)}$ is the estimated value of $\beta$ in ith iteration. To compare the performances of the estimators, percentage relative efficiency of the $r-(k, d)$ class estimator over the PCTP estimator has been calculated as follows:

$$
\% \mathrm{RE}=\frac{\operatorname{EMSE}\left(\widetilde{\beta}_{r}(k, d)\right)-\operatorname{EMSE}\left(\widehat{\beta}_{r}(k, d)\right)}{\operatorname{EMSE}\left(\widehat{\beta}_{r}(k, d)\right)} \times 100
$$

For brevity, we have reported some selected results in Tables 1-3, where $E_{1}, E_{2}$, and $E_{3}$ give percent relative efficiency of the $r-(k, d)$ class estimator over the PCTP estimator when $\gamma$ takes values $0.90,0.95$, and 0.99 , respectively.

Since all the values of percent relative efficiency shown in Tables 1-3 are positive, it implies that the $r-(k, d)$ class estimator is more efficient than the PCTP estimator in all the cases considered in this study. However, when we examine the behavior of the percent relative efficiency for different parameters considered here, it is observed that the parameters $n, p, \rho$, and $\gamma$ affect the percent relative efficiency negatively. That is, when these parameters take larger values, the percent relative efficiency decreases and approaches zero. Alternatively, we can say that there are some values of the parameters for which the value of percent relative efficiency is so low that it can be considered that both the estimators perform equally well, for example, when $\sigma_{e}^{2}=0.1, n=$ $20,50,100$, and $p=10$ and $\sigma_{e}^{2}=0.1, n=20,50,100, p=5$, and $\rho=0.9$ for most of the values of $k$ and $d$.

\section{Numerical Example}

In this section we examine the performance of the two estimators, namely, the $r-(k, d)$ class estimator and the PCTP estimator in MSE sense using two numerical examples; one is for US GDP data and the other is the famous Hald data [33].

Example 1 (US GDP Data). The quarterly US data on GDP growth $(y)$, personal disposable income $\left(X_{1}\right)$, personal consumption expenditure $\left(X_{2}\right)$, and corporate tax after profits $\left(X_{3}\right)$ for the years 1970-1991 have been taken from Gujarati [34]. The data has also been used by Chandra and Sarkar [24]. The variables are standardized and the eigenvalues of $X^{\prime} X$ matrix are obtained as 324.6527396, 21.8742084, 1.2365875, and 0.2364645 , which shows high multicollinearity in the data. Further, the value of the DW statistic for the data is found to be 0.4784 , which indicates the presence of positive autocorrelation at the significance level of 0.05 with the two limits of the critical value being $d_{L}=1.429$ and $d_{U}=$ 1.611 for $n=88$. The error structure follows $\operatorname{AR}(1)$ process with estimated $\rho$ to be 0.7530 and $\sigma_{e}^{2}=0.001118$. Thus the $\Omega$ matrix can be constructed using (28). The condition number of $X^{\prime} \Omega^{-1} X$ is obtained to be 246.3951 which indicates high multicollinearity in the data. Although the GLSE is unstable in the presence of multicollinearity, several studies have suggested that the estimate of variance based on least squares estimator is superior to the estimates based on other shrinkage estimators, for instance, see Ohtani [35], Dube and Chandra [36], and Ünal [37]. Hence, we have estimated the value of $\sigma^{2}$ by using the GLSE of $\beta$, which comes out to be 0.00292. The eigenvalues of $X^{\prime} \Omega^{-1} X$ are $\lambda_{1}=23.78081$, $\lambda_{2}=3.0276, \lambda_{3}=0.2420$, and $\lambda_{4}=0.0965$. We chose $r=2$ which accounts for $99.57 \%$ of variation in the data. Now, we chose a value of $k$ to be 0.2505 such that $k>\sigma^{2} / \alpha_{\min }^{2}=0.1505$ for the $r-(k, d)$ class estimator and thus the optimum value $d_{1_{\text {opt }}}$ is obtained to be 0.4286 . Further, since estimated $\sigma^{2}$ is smaller than $\alpha_{r}^{2}=(0.258642223,0.0194366), k$ is selected as 0.0056 which belongs to the interval $0<k<\sigma^{2} / \alpha_{\max }^{2}=$ 0.01131 and hence $d_{2_{\text {opt }}}$ is found to be 0.8501 . The MSEs of the $r-(k, d)$ class estimator and the PCTP estimator for the obtained optimum values are 0.1396499 and 0.1396482 , respectively. Clearly, the values are almost the same which suggests that both the estimators perform equally well for the corresponding optimum values. However, the difference can 


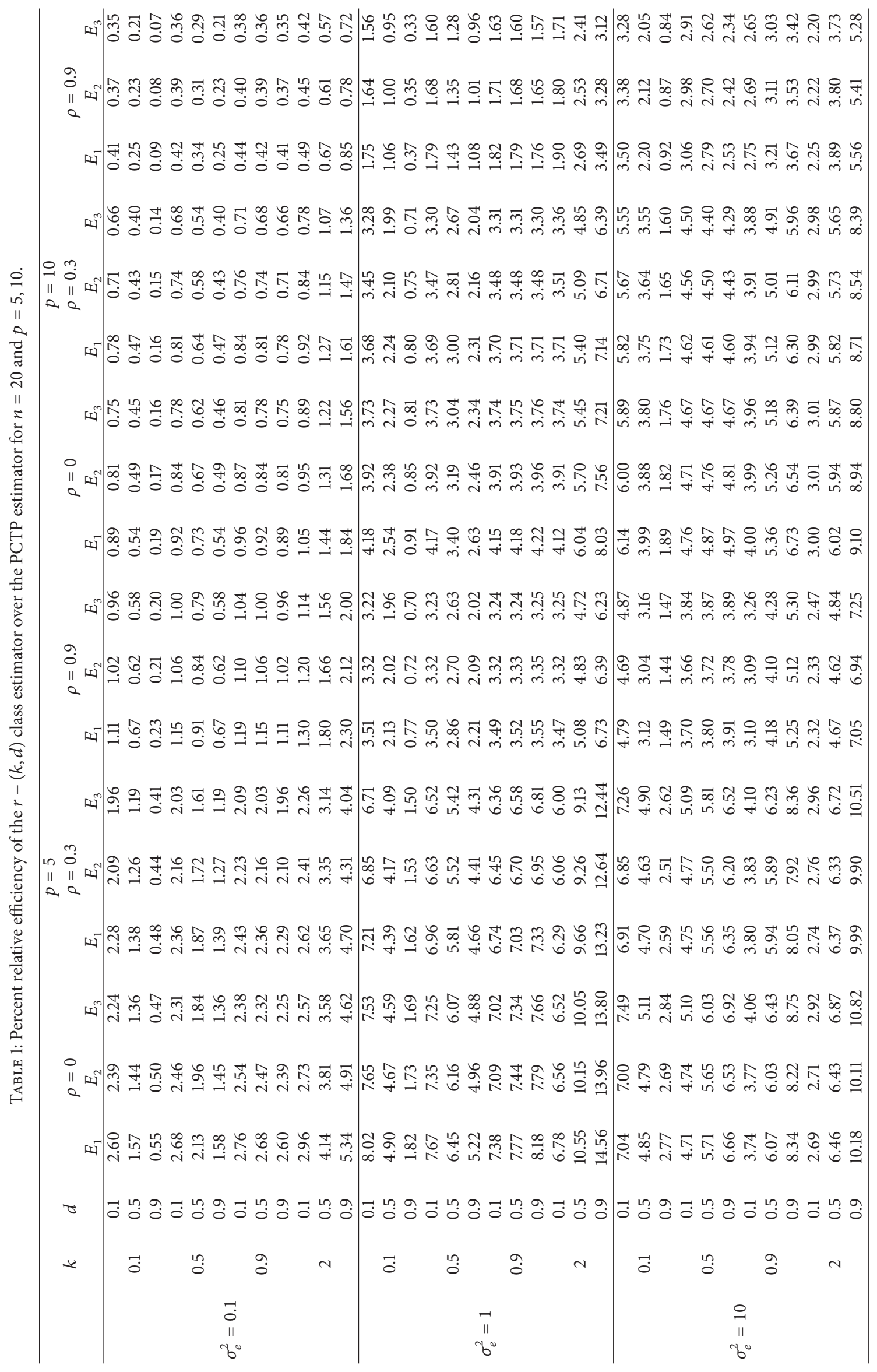




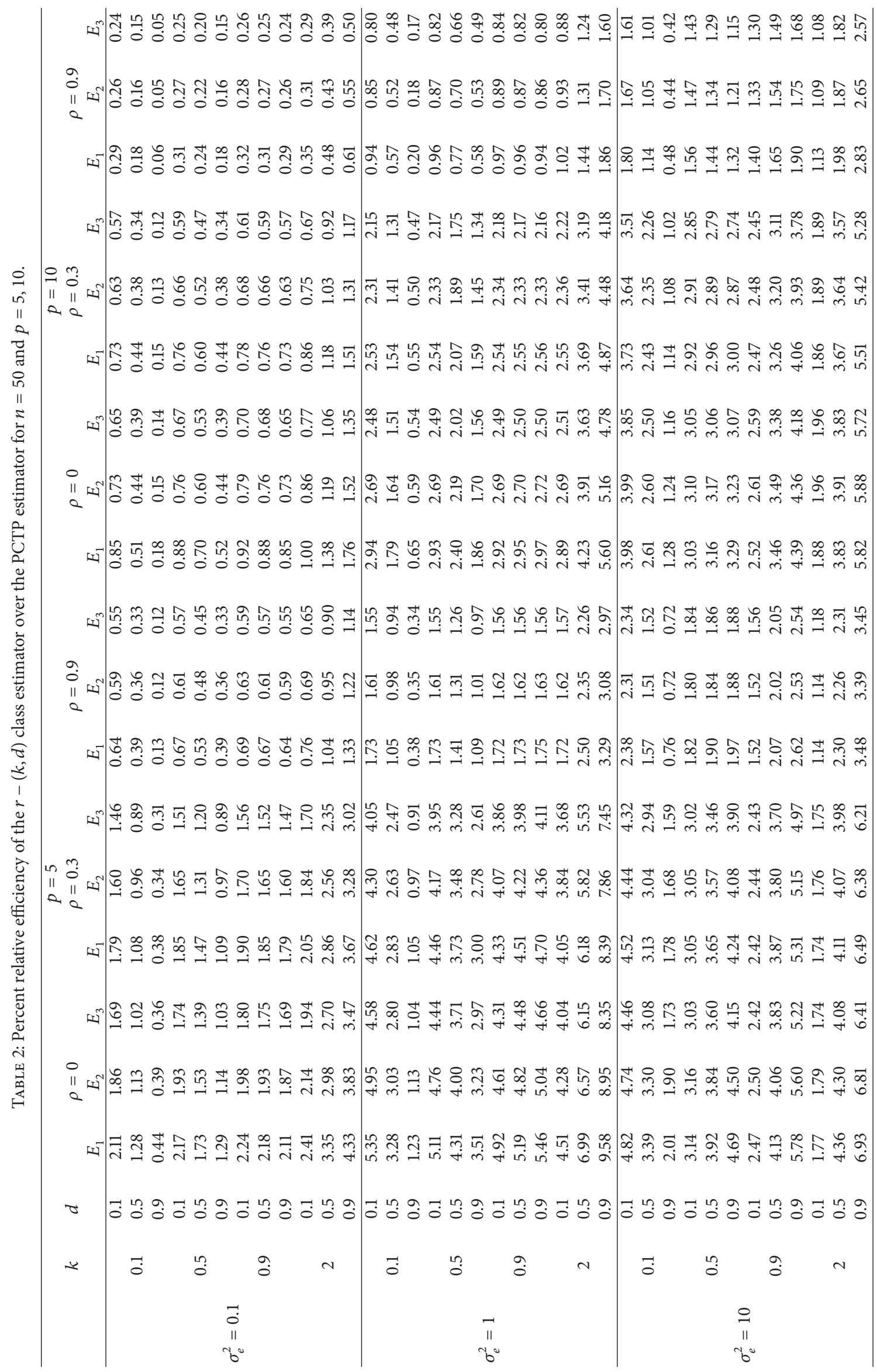




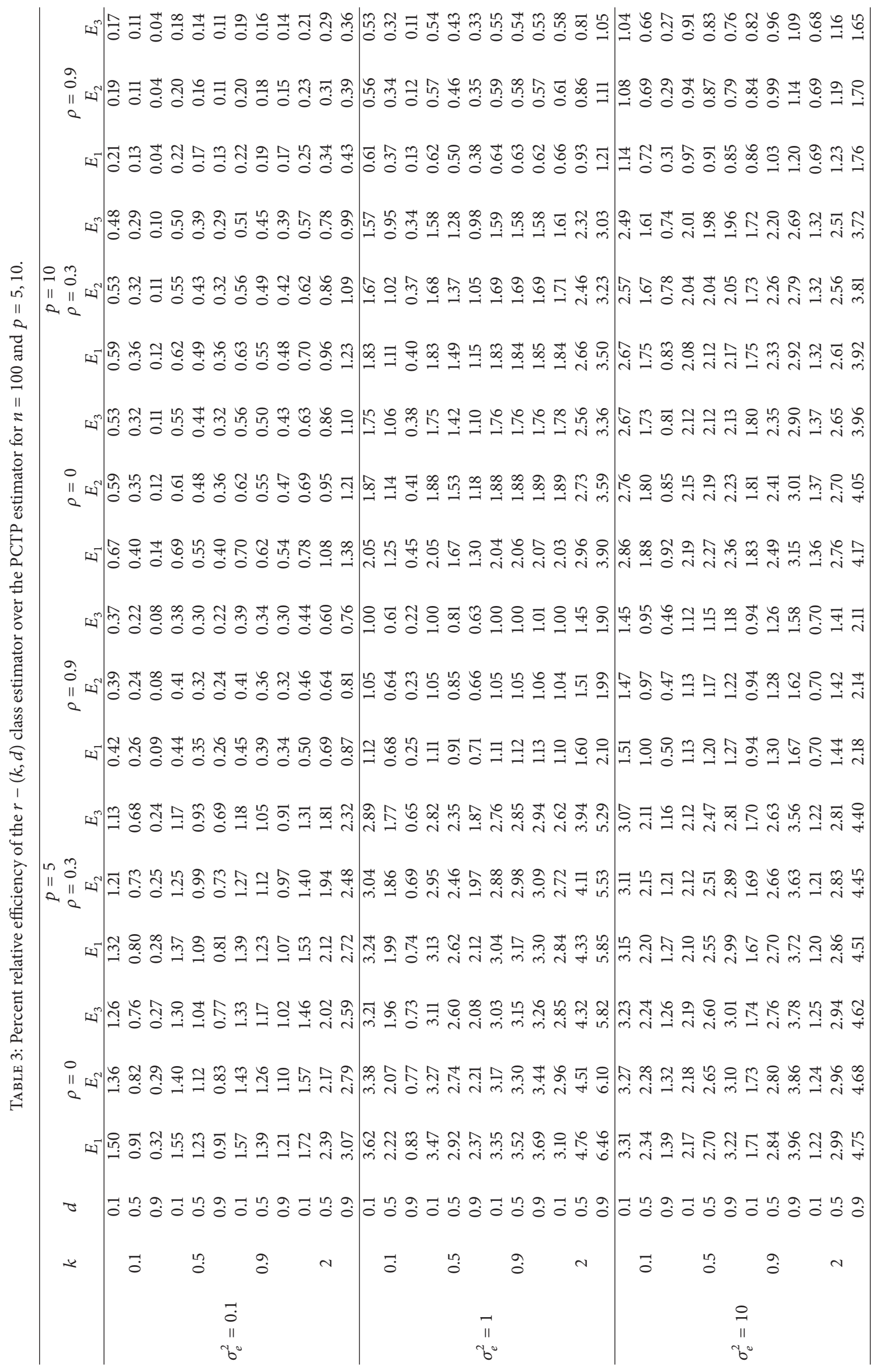




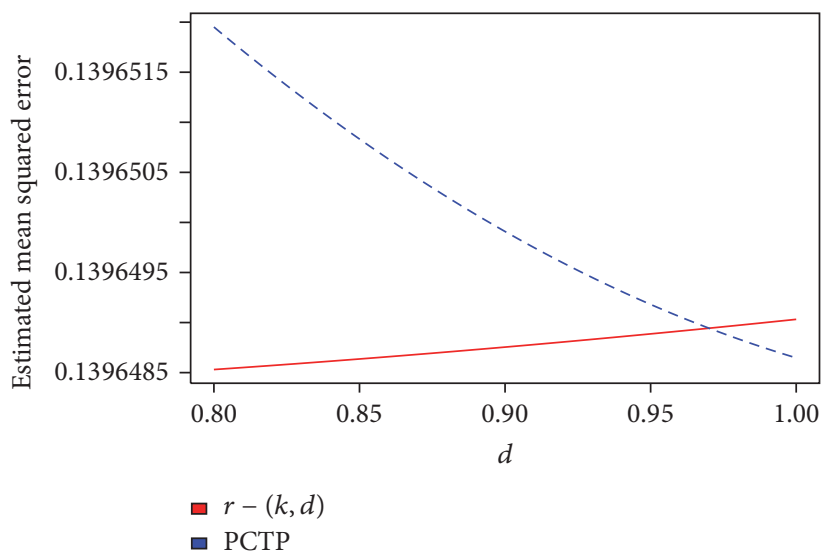

(a) $k=0.25$

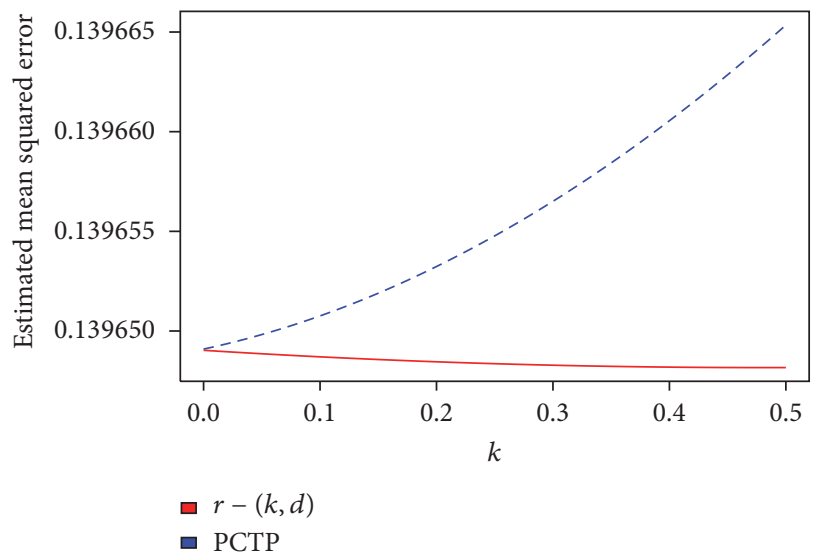

(c) $d=0.7$

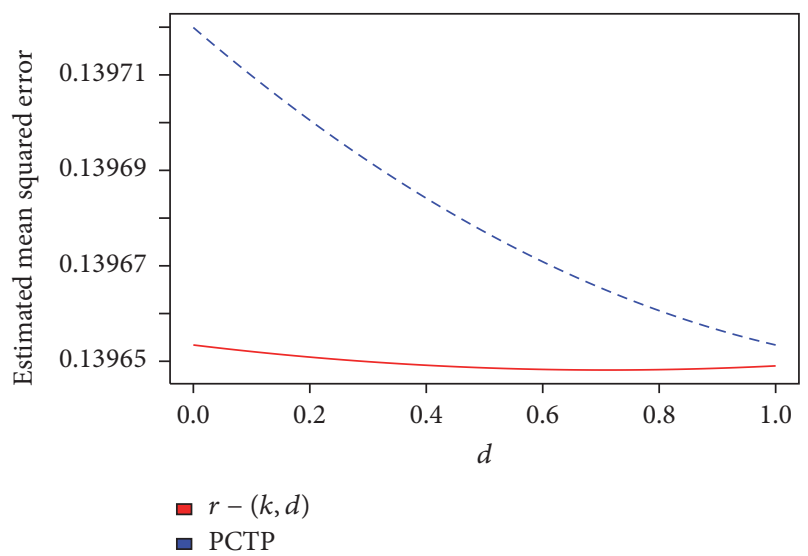

(b) $k=0.5$

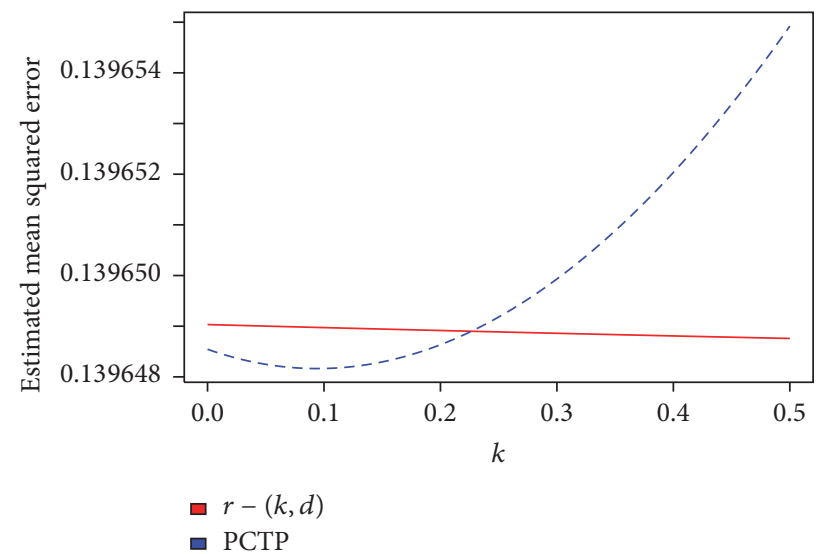

(d) $d=0.95$

FIGURE 1: Estimated mean squared error of $r-(k, d)$ class and the PCTP estimators for US GDP data.

be noticed after 5 decimal places, indicating superiority of the PCTP estimator over the $r-(k, d)$ class estimator in MSE sense.

Further, the estimated MSEs of the $r-(k, d)$ class and the PCTP estimators with respect to $d$ for fixed $k=0.25,0.5$ and with respect to $k$ for $d=0.7,0.95$ are represented in Figure 1 . The values of $k$ and $d$ are selected so that we can observe the behavior of estimators for $k$ and $d$ near and far from their respective optimum values. The figures depict superiority of the $r-(k, d)$ estimator over the PCTP estimator for larger range of $k$ and $d$. Further, it can be seen from Figure 1(a) that the PCTP estimator starts dominating the $r-(k, d)$ class estimator after a point, whereas for $k=0.5$ in Figure 1(b) we do not see a superiority of the PCTP estimator in the whole range of $0<d<1$. Similarly, we get a range of $k$ in which the PCTP estimator dominates the $r-(k, d)$ class estimator for $d=0.95$ and not for $d=0.7$; see Figures 1(c) and 1(d).

Example 2 (Hald Data). Now, let us consider the data set on Portland cement originally due to Woods et al. [38] and then analyzed by Hald [33] and known as Hald data. The data is an outcome of an experiment conducted to investigate the heat evolved during setting and hardening of Portland cements of varied composition and the dependence of this heat on the percentages of four compounds in the clinkers from which the cement was produced. The data includes the heat evolved in calories per gram of cement $(y)$ as dependent variable and four ingredients: tricalcium aluminate $\left(X_{1}\right)$, tetracalcium silicate $\left(X_{2}\right)$, tetracalcium aluminoferrite $\left(X_{3}\right)$, and dicalcium silicate $\left(X_{4}\right)$ as explanatory variables.

Following Özkale [11], the variables are standardized so that the $X^{\prime} X$ matrix forms a correlation matrix and the eigenvalues obtained are $\lambda_{1}=2.235704, \lambda_{2}=1.576066, \lambda_{3}=$ 0.186606 , and $\lambda_{4}=0.001623$. The value of Durbin-Watson test comes out to be 2.052597 resulting in the conclusion of no autocorrelation in error term at $5 \%$ level of significance with $d_{L}=0.574$ and $d_{U}=2.094$ for $n=13$; hence we can consider $\Omega=I_{n}$. The estimated value of $\sigma^{2}$ is 0.00196 and the value of $r$ has been chosen to be 2, which accounts for $95.29 \%$ of variation in data. The optimum value of $d$ for a selected value of $k$ is chosen for the $r-(k, d)$ class and PCTP estimators. For the $r-(k, d)$ class estimator, we chose value of $k$ as 1.322 such that $k>\sigma^{2} / \alpha_{\min }^{2}=1.222$, and for this value of $k$ the optimum value $d_{1_{\text {opt }}}$ is obtained as 0.9899 . Further, we obtain $\alpha_{r}^{2}=(0.4348143,0.0016061)$ which indicates that $\sigma^{2}$ is larger than the second value of $\alpha_{r}^{2}$, 


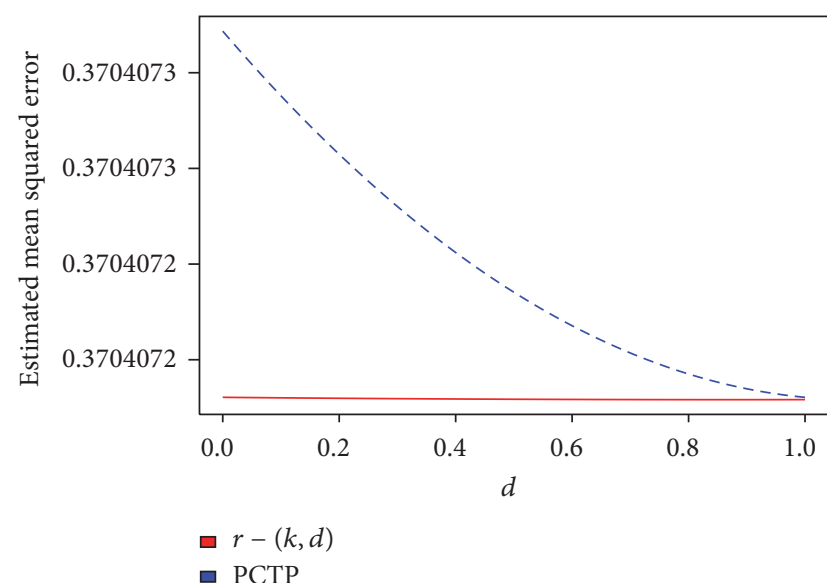

(a) $k=0.1$

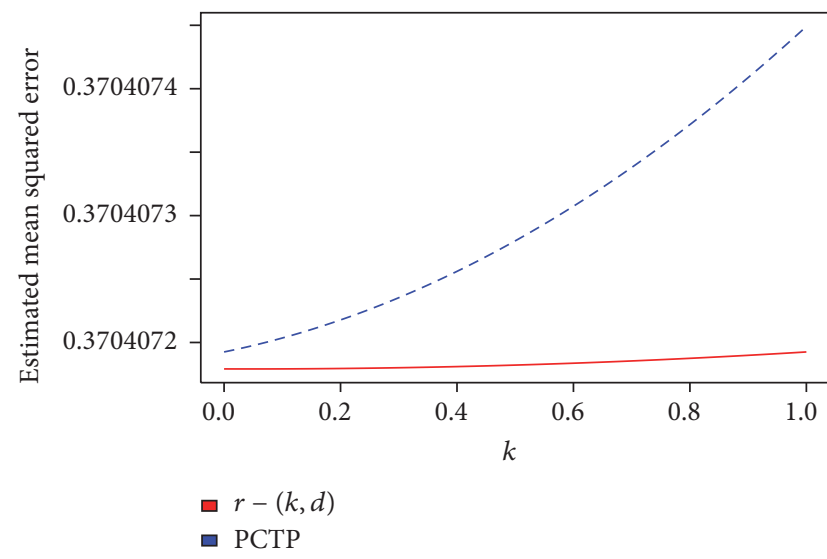

(c) $d=0.7$

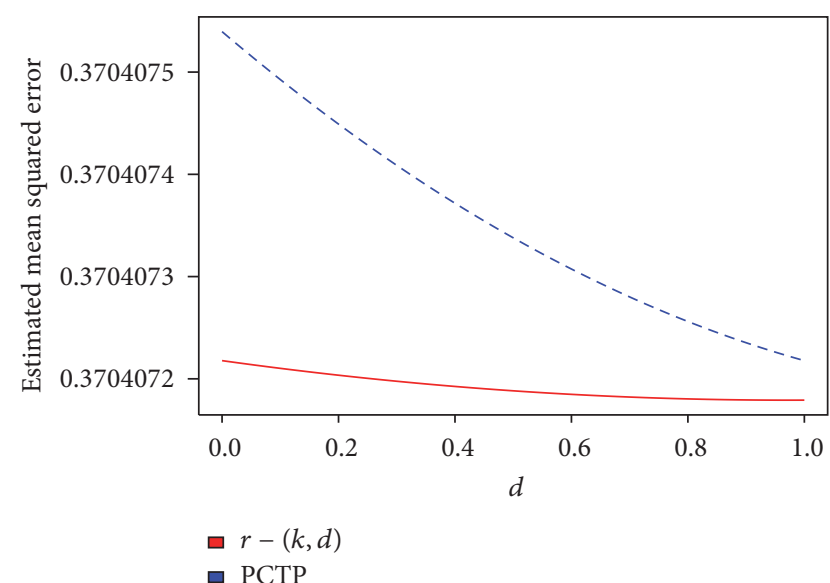

(b) $k=0.5$

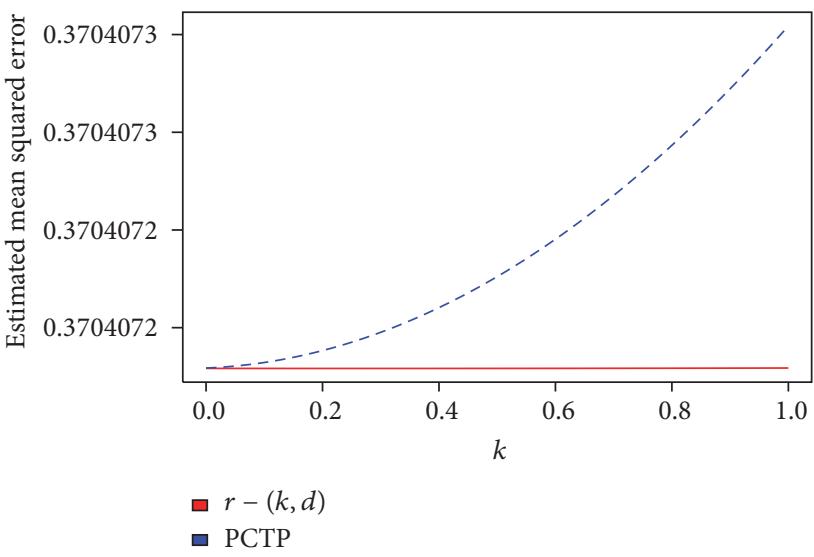

(d) $d=0.95$

FIGURE 2: Estimated mean squared error of $r-(k, d)$ class and the PCTP estimators for Hald data.

hence we chose a value of $k$ in the range $\max _{i=1,2, \ldots, r}\left\{\lambda_{i}\left(\sigma^{2}-\right.\right.$ $\left.\left.\alpha_{i}^{2}\right) /\left(\lambda_{i}+1\right) \alpha_{i}^{2}\right\}<k<\sigma^{2} / \alpha_{\max }^{2}$. The value of lower bound is obtained as 0.000000574 , which is approximately 0 and the upper bound is 0.004516 ; hence we chose a value $k$ as 0.0022 and for this value $d_{2_{\text {opt }}}$ for the PCTP estimator is obtained as 0.9854 . The MSEs of the $r-(k, d)$ class and PCTP estimators are obtained to be the same up to 7 decimal places, that is, 0.3704072 , indicating the same performance at the optimum values.

Moreover, the performance of both estimators for various $k$ and $d$ is represented in Figures 2(a)-2(d). Clearly, the $r-(k, d)$ class estimator is exhibiting better performance for larger range of $k$ and $d$. However, a careful examination of Figures 2(a) and 2(d) suggests that there may be some points of $k$ and $d$ where the PCTP may perform better. Figures 2(b) and 2(c) show that there is no value of $d$ and $k$, respectively, for $k=0.5$ and $d=0.7$.

Looking at the results of both examples we observe that the $r-(k, d)$ class estimator performs better than the PCTP estimator in scalar MSE sense for most of the values of $k$ and $d$ under study. However, Figures 1(a), 1(d), 2(a), and 2(d) are showing a possibility of superiority of the PCTP estimator over the $r-(k, d)$ class estimator.

\section{Conclusion}

In this paper we have examined the performance of two biased estimators in the presence of multicollinearity with autocorrelated errors which include the same number of unknown parameters with same range. Further, a method of selection of $k$ and $d$ in both estimators has been suggested in terms of minimizing scalar MSE. The conditions of dominance of the PCTP estimator over the $r-(k, d)$ class estimator have been derived using MSE matrix as comparison criterion. Further, we have performed a simulation study and the percentage relative efficiency of the $r-(k, d)$ class estimator over the PCTP estimator has been evaluated. Moreover, two numerical examples are considered to compare the two estimators. The simulation study suggests that for all the parametric conditions considered here the $r-(k, d)$ class estimator performs better than the PCTP estimator in scalar MSE sense. The numerical examples give the results in favor of the simulation results; that is, the $r-(k, d)$ class estimator performed better than the PCTP estimator under scalar MSE criterion for most of the values of $k$ and $d$. However, for optimum values of $k$ and $d$ the performance of both estimators is similar and the superiority of the PCTP estimator may be seen after the fourth or fifth decimal places. 


\section{Competing Interests}

There are no conflicts of interest regarding the publication of this paper.

\section{References}

[1] C. Stein, "Inadmissibility of usual estimator for the mean of a multivariate normal distribution," in Proceedings of the $3 \mathrm{rd}$ Berkeley Symposium on Mathematical Statistics and Probability, pp. 197-206, University of California Press, 1956.

[2] A. E. Hoerl and R. W. Kennard, "Ridge regression: applications to nonorthogonal problems," Technometrics, vol. 12, no. 1, pp. 69-82, 1970.

[3] A. E. Hoerl and R. W. Kennard, "Ridge regression: biased estimation for nonorthogonal problems," Technometrics, vol. 12, no. 1, pp. 55-67, 1970.

[4] W. F. Massy, "Principal components regression in exploratory statistical research," Journal of the American Statistical Association, vol. 60, no. 309, pp. 234-256, 1965.

[5] M. R. Baye and D. F. Parker, "Combining ridge and principal component regression: a money demand illustration," Communications in Statistics - Theory and Methods, vol. 13, no. 2, pp. 197-205, 1984.

[6] M. Nomura and T. Ohkubo, "A note on combining ridge and principal component regression," Communications in Statistics. Theory and Methods, vol. 14, no. 10, pp. 2473-2487, 1985.

[7] K. J. Liu, "A new class of biased estimate in linear regression," Communications in Statistics. Theory and Methods, vol. 22, no. 2, pp. 393-402, 1993.

[8] S. Kaçiranlar and S. Sakallığlu, "Combining the liu estimator and the principal component regression estimator," Communications in Statistics-Theory and Methods, vol. 30, no. 12, pp. 2699-2705, 2001.

[9] M. R. Özkale and S. Kaçıranlar, "The restricted and unrestricted two-parameter estimators," Communications in StatisticsTheory and Methods, vol. 36, no. 15, pp. 2707-2725, 2007.

[10] H. Yang and X. Chang, "A new two-parameter estimator in linear regression," Communications in Statistics-Theory and Methods, vol. 39, no. 6, pp. 923-934, 2010.

[11] M. R. Özkale, "Combining the unrestricted estimators into a single estimator and a simulation study on the unrestricted estimators," Journal of Statistical Computation and Simulation, vol. 82, no. 5, pp. 653-688, 2012.

[12] X. Chang and H. Yang, "Combining two-parameter and principal component regression estimators," Statistical Papers, vol. 53, no. 3, pp. 549-562, 2012.

[13] A. C. Aitken, "IV.—on least squares and linear combination of observations," Proceedings of the Royal Society of Edinburgh, vol. 55, pp. 42-48, 1936.

[14] G. Trenkler, "On the performance of biased estimators in the linear regression model with correlated or heteroscedastic errors," Journal of Econometrics, vol. 25, no. 1-2, pp. 179-190, 1984.

[15] L. L. Firinguetti, "A simulation study of ridge regression estimators with autocorrelated errors," Communications in Statistics. Simulation and Computation, vol. 18, no. 2, pp. 673-702, 1989.

[16] G. M. Bayhan and M. Bayhan, "Forecasting using autocorrelated errors and multicollinear predictor variables," Computers and Industrial Engineering, vol. 34, no. 2-4, pp. 413-421, 1998.
[17] M. I. Alheety and B. M. G. Kibria, "On the liu and almost unbiased liu estimators in the presence of multicollinearity with heteroscedastic or correlated errors," Surveys in Mathematics and its Applications, vol. 4, pp. 155-167, 2009.

[18] M. R. Özkale, "Principal components regression estimator and a test for the restrictions," Statistics. A Journal of Theoretical and Applied Statistics, vol. 43, no. 6, pp. 541-551, 2009.

[19] H. Güler and S. Kaçiranlar, "A comparison of mixed and ridge estimators of linear models," Communications in Statistics: Simulation and Computation, vol. 38, no. 2, pp. 368-401, 2009.

[20] M. A. Alkhamisi, "Ridge estimation in linear models with autocorrelated errors," Communications in Statistics. Theory and Methods, vol. 39, no. 14, pp. 2630-2644, 2010.

[21] H. Yang and J. Wu, "Estimation in singular linear models with stochastic linear restrictions and linear equality restrictions," Communications in Statistics-Theory and Methods, vol. 40, no. 24, pp. 4364-4371, 2011.

[22] H. Y. A. Eledum and A. A. Alkhalifa, "Generalized two stages ridge regression estimator GTR for multicollinearity and autocorrelated errors," Canadian Journal on Science and Engineering Mathematics, vol. 3, pp. 79-85, 2012.

[23] G. Şiray, S. Kaçiranlar, and S. Sakallıoğlu, "r-k class estimator in the linear regression model with correlated errors," Statistical Papers, vol. 55, no. 2, pp. 393-407, 2014.

[24] S. Chandra and N. Sarkar, "A restricted r-k class estimator in the mixed regression model with autocorrelated disturbances," Statistical Papers, vol. 57, no. 2, pp. 429-449, 2016.

[25] S. Chandra and G. Tyagi, "A general class of biased estimators in the presence of multicollinearity with autocorrelated errors," International Journal of Mathematics and Statistics, vol. 18, no. 2, 2017.

[26] J. Huang and H. Yang, "On a principal component twoparameter estimator in linear model with autocorrelated errors," Statistical Papers, vol. 56, no. 1, pp. 217-230, 2015.

[27] A. Hoerl, R. Kennard, and K. Baldwin, "Ridge regression: some simulations," Communication in Statistics, vol. 4, pp. 105-123, 1975.

[28] B. M. Kibria, "Performance of some new ridge regression estimators," Communications in Statistics. Simulation and Computation, vol. 32, no. 2, pp. 419-435, 2003.

[29] G. C. McDonald and D. I. Galarneau, "A monte carlo evaluation of some ridge-type estimators," Journal of the American Statistical Association, vol. 70, no. 350, pp. 407-416, 1975.

[30] D. G. Gibbons, "A simulation study of some ridge estimators," Journal of the American Statistical Association, vol. 76, no. 373, pp. 131-139, 1981.

[31] G. Judge, W. Griffiths, R. Hill, H. Lütkepohl, and T. Lee, The Theory and Practice of Econometrics, John Wiley \& Sons, New York, NY, USA, 1985.

[32] R. A. Johnson and D. W. Wichern, Applied Multivariate Statistical Analysis, Pearson-Prentice Hall, New York, NY, USA, 2007.

[33] A. Hald, Statistical Theory with Engineering Applications, John Wiley \& Sons, New York, NY, USA, 1952.

[34] D. N. Gujarati, Basic Econometrics, Mc-Graw Hill, New York, NY, USA, 2002.

[35] K. Ohtani, "Inadmissibility of the iterative Stein-rule estimator of the disturbance variance in a linear regression," Economics Letters, vol. 24, no. 1, pp. 51-55, 1987.

[36] M. Dube and S. Chandra, "A note on inadmissibility of the iterative stein-rule estimator of the disturbance variance," Journal of 
the Indian Society of Agricultural Statistics, vol. 61, pp. 341-343, 2007.

[37] D. Ünal, "The effects of the proxy information on the iterative Stein-rule estimator of the disturbance variance," Statistical Papers, vol. 51, no. 2, pp. 477-484, 2010.

[38] H. woods, H. H. Steinour, and H. R. Starke, "Effect of composition of portland cement on heat evolved during hardening," Industrial \& Engineering Chemistry, vol. 24, no. 11, pp. 1207-1214, 1932. 


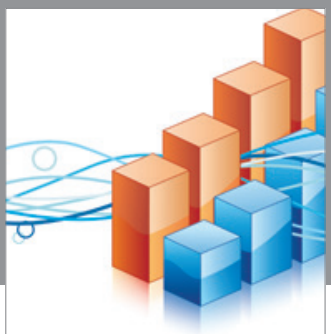

Advances in

Operations Research

vatem alat4

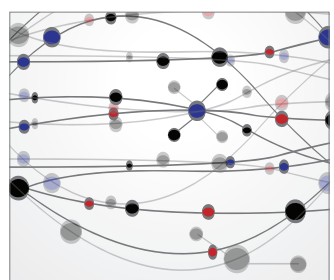

\section{The Scientific} World Journal
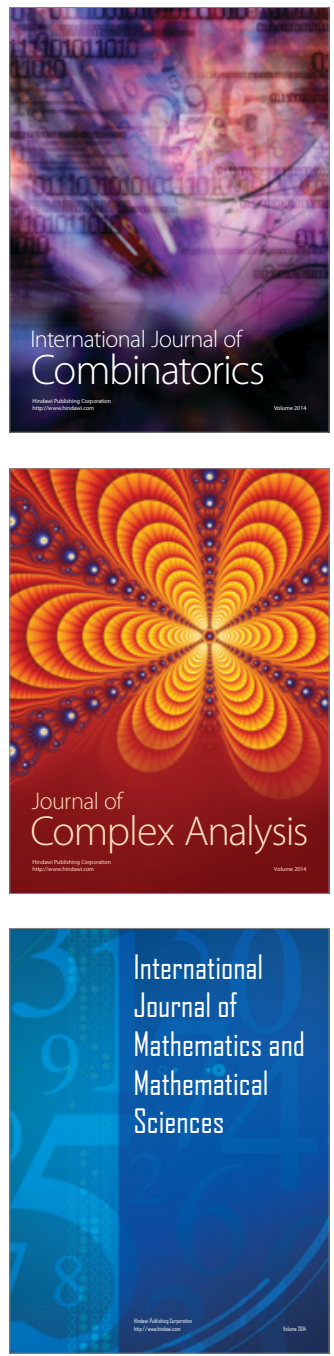
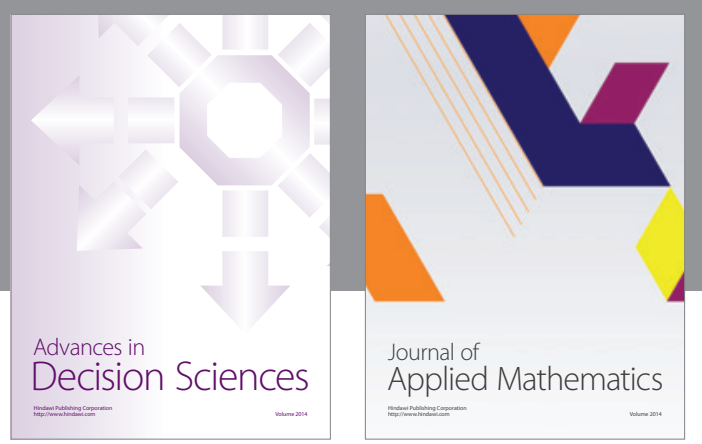

Algebra

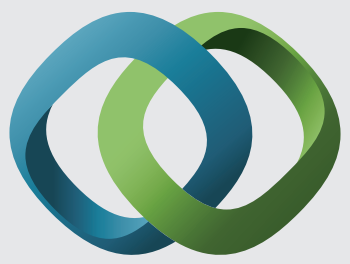

\section{Hindawi}

Submit your manuscripts at

https://www.hindawi.com
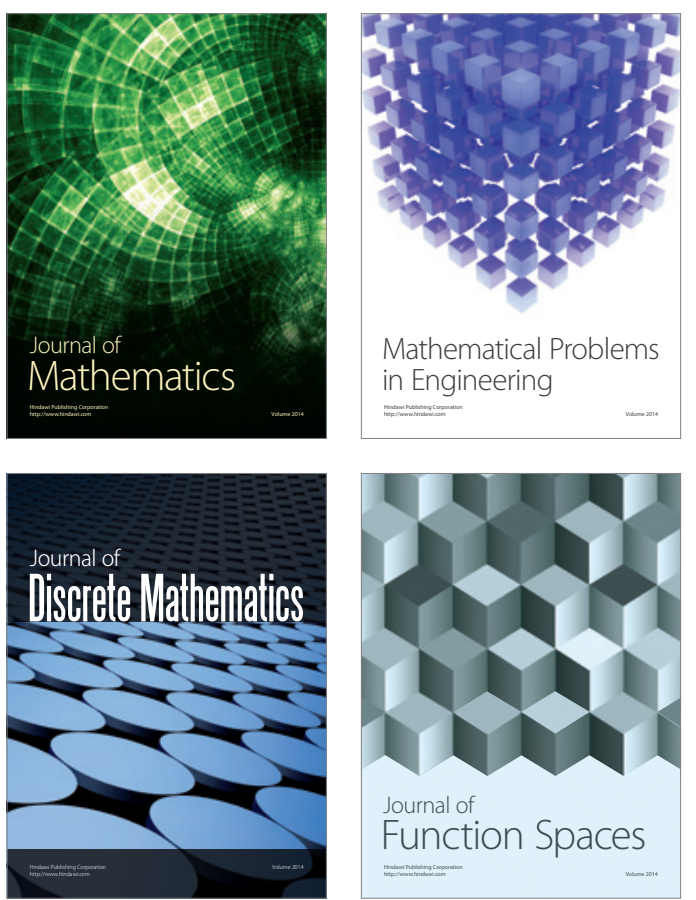

Mathematical Problems in Engineering


Journal of

Function Spaces

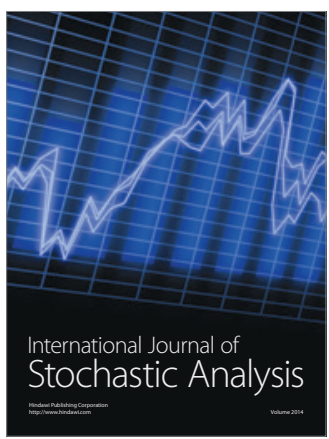

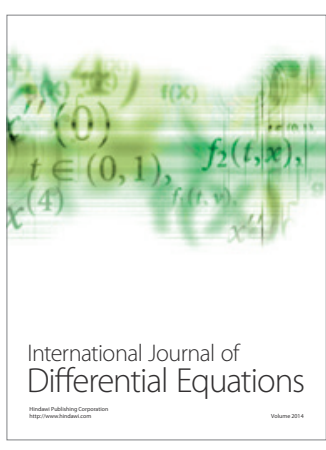
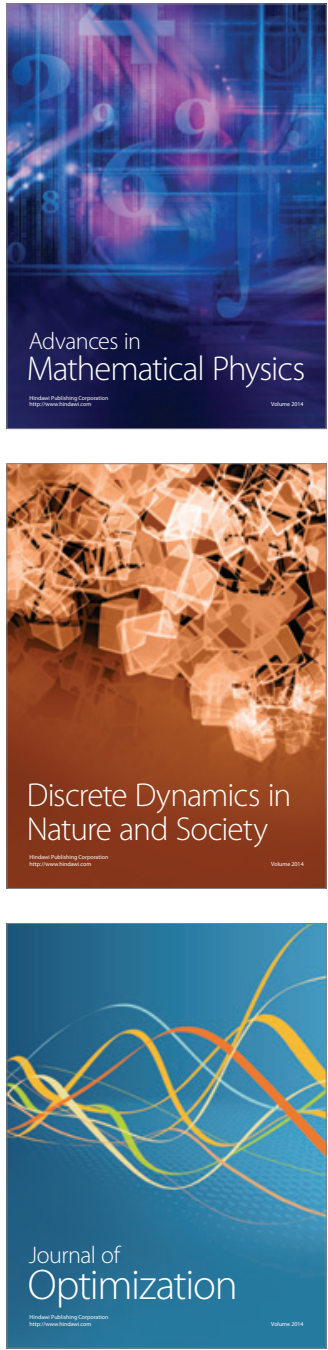